\title{
Variation in growth rate in a natural assemblage of unicellular green soil algae
}

\author{
HANS P. KOELEWIJN*, PATRICK DE LA GUERIE $\$$ \& GRAHAM BELL \\ Redpath Museum and Department of Biology, McGill University, 859 Sherbrooke Street West, Montreal, Quebec, \\ Canada, H3A 2K6 and \$Laboratoire de Génétique et Évolution des populations vegetales, UPRESA CNRS 8016, \\ Bâtiment SN2, Université de Lille I, 59655 Villeneuve d'Asq Cedex, France
}

\begin{abstract}
Unicellular, motile, phototropic green algae were extracted from soil samples taken at metre intervals along a $25-\mathrm{m}$ transect in a wheat field. The vegetative growth of 61 randomly selected isolates (henceforth called spores) was measured in dark and light conditions, and at high and low nutrient concentrations in liquid media. The among-spore variance was dominated by a spore-by-environment interaction. The increase of among-spore variance with distance was detectable but slight, showing that most of the diversity found on the transect was present at a scale of metres. A mixture of all spores, constituting the founder assemblage, was propagated for 50-70 generations in three environments to study the sorting of variation initially present in the assemblage. Adaptation to the new environments was measured by improvement in growth relative to the founder assemblage. All three sets of lines became adapted to their selection environment, although the extent of the advance depended on the amount of among-spore variance in the founder assemblage. Selection for increased growth in the dark, or at high nutrient conditions in the light, caused the assemblage to evolve to the limit of the variation initially present, strongly reducing among-spore variance as a consequence. At low nutrient concentrations selection was less effective, and there was little reduction of the amongspore variance. The correlated responses of each group, again relative to the founder assemblage, were measured over the entire experimental range. Correlated responses were negative when performance in widely different (light and dark) environments was compared, but positive for more similar environments (high and low nutrient concentrations).
\end{abstract}

Keywords: Chlamydomonas, competition, environmental heterogeneity, genetic diversity, soil microbes, sorting.

\section{Introduction}

One of the central problems in biology is the maintenance of diversity. Ecologists have been impressed by the vast number of species coexisting in a small area; geneticists by the range of variation within species or populations. In either case, environmental heterogeneity has been suggested as one of the prime causes for the maintenance of this diversity (Levene, 1953; Levins, 1968; Hedrick, 1986; Gillespie \& Turelli, 1989; Bell, 1997). In an uniform environment, one might expect that the type that reproduced fastest would replace all others. In an heterogeneous environment, however, a variety of types might exist if each were superior to all others in a particular place or in a particular range of conditions (i.e. type-by-environment interaction). Rosenzweig (1995)

*Correspondence and present address: Netherlands Institute of Ecology, PO Box 40, Boterhoeksestraat 48, 6666 ZG Heteren, The Netherlands. E-mail: koelewijn@cto.nioo.knaw.nl has summarized the case for believing that species diversity can be understood largely in terms of habitat diversity. At the level of genetic variation within populations, the evidence is not as clear (Hedrick, 1986).

The heterogeneity of the environment can be expressed on a temporal and a spatial scale. For plants, for example growing in a certain area, the temporal variability caused by the weather will usually be the same. It is unlikely, however, that they all experience the same growing conditions, because of spatial variation of the substrate. Environments are rarely homogeneous, even at small spatial scales (Ball \& Williams, 1968; Beckett \& Webster, 1971; Westerman, 1990). Several researchers have found variation at very small scales (up to $0.1 \mathrm{~m}$ ) for physical or chemical characteristics of soil in natural systems (Taylor et al., 1982; Troelstra et al., 1990; Lechowicz \& Bell, 1991). Whether an organism will also perceive this abiotic variation, and thus experience the environment as either coarse-grained or fine-grained (Levins, 1968) or homogeneous or 
heterogeneous, depends on its dispersal capacities. If dispersal is limited and the environment coarse-grained, offspring will be more likely to grow in conditions similar to those experienced by their parents, so that adaptation to the specific sites can evolve (Antonovics \& Levin, 1980; Heywood, 1991). In a fine-grained or heterogeneous environment, offspring will more likely grow up in different conditions at different sites, so that adaptation is less likely to occur.

If the diversity of types in natural populations or assemblages is maintained primarily through a typeby-environment interaction, then different types will be selected in different combinations of physical and chemical conditions. Culturing the population or assemblage in a defined environment will then cause adaptation to that environment, accompanied by the loss of variance in growth rate. The first stage in this process will be the sorting of the variation originally present in the population. Those types which happen to be better adapted to the new environment will tend to increase in frequency. As selection sorts the initial population the mean growth rate of the population as a whole must increase continuously. In this way, the population will eventually come to consist almost entirely of one of the types that have the highest growth rate in the changed conditions. The population has reached its sorting limit and exhausted the variance in growth rate initially present. The next stage to increase adaptedness will be through the selection of newly originated variants with slightly higher growth rates, caused by mutation (Lenski \& Travisano, 1994; Kibota \& Lynch, 1996; Bell \& Reboud, 1997). This will bring the population beyond the sorting limit of existing variation. Besides increased adaptedness, sorting or continued selection of types might also lead to a negative correlation between growth in strongly contrasted environments, because adaptation to one environment should sometimes obstruct adaptation to another environment (Bell \& Reboud, 1997).

The study of the evolutionary process of adaptation to novel environments requires long-term selection studies with large populations. These studies are laborious and often scarcely feasible, either with plants or with animals. Microbes, however, offer significant advantages for experimental studies of evolutionary processes. They can be maintained under defined culture conditions with enormous population sizes for hundreds of generations. Experimental lines may be replicated so that rigorous statistical analyses of the processes involved are possible. Finally, control over experimental conditions permits comparison of evolution in uniform and heterogeneous environments (Reboud \& Bell, 1997). Starting from isogenic lines, the bacterium Escherichia coli and the chlorophyte Chlamydomonas reinhardtii have now been used to study the process of adaptation to novel environments and the occurrence of adaptive divergence (Bennett et al., 1992; Bennett \& Lenski, 1993; Travisano et al., 1995; Bell \& Reboud, 1997). The stage before arriving at isogenic lines, i.e. the sorting of the variation initially present, however, has received less attention.

Here we report the magnitude of a type-by-environment interaction and the sorting of variation in novel environments of an assemblage of unicellular, motile, phototropic green algae, freshly extracted from soil samples along a $25-\mathrm{m}$ transect. The assemblage consisted of 61 randomly selected isolates and represents part of the algal community present in the soil. We describe the properties of this assemblage in terms of the vegetative (asexual) growth of the isolates under different environmental conditions and compare the properties of the assemblage before and after selection. We are not concerned with the taxonomic status of the algae, but rather with the phenotypic community response to selection. The main object of our study was to analyse the diversity of this community of soil algae through its response to different conditions of culture, enabling us to measure both the rate and the extent of sorting, and to compare direct and correlated responses to selection.

\section{Materials and methods}

\section{Collection of material}

Two soil samples of about $0.5 \mathrm{~cm}^{3}$, each $10 \mathrm{~cm}$ apart, were taken every metre along a $25-\mathrm{m}$ transect in a wheat field near Farnham, Quebec. The choice of spatial scale was to some extent arbitrary, but we had previously shown that considerable variation can be found even at smaller scales (unpublished observations). Algae were extracted from these samples using the method of Sack et al. (1994), which selects for green, unicellular, motile, phototropic algae resembling Chlamydomonas. The locality used has yielded many isolates of $C$. reinhardtii, but none of the spores in this series mated with standard laboratory strains of this species. Chlamydomonas is a diverse genus of unicellular green algae, which lives mostly in soils. It is usually asexual, so that genotypes are readily replicated and stored. It is a photoautotroph that is easily cultured in axenic, chemically defined media of simple inorganic salts, allowing straightforward and repeatable manipulation of environments. Because we were selecting Chlamydomonas-like algae, the algae were stored and grown according to the protocols used for Chlamydomonas (Harris, 1989). The number of isolates extracted from a sample ranged from 0 to 23 . From each sample which yielded algae two isolates (henceforth called spores) were chosen randomly, giving a total of 61 axenic cultures, which were 
stored on agar slants. These cultures represent part of the community of soil algae and may represent different species and different genotypes within species. Their taxonomic status is unknown, but is not relevant to the aim of our study which was to look for the short-term response of the phenotypic characteristics of the algae community to selection. The cultures were tested for the production of gametes and zygotes, according to Chlamydomonas protocols, but none were observed.

\section{Initial diversity}

We grew cultures in modifications of Bold's medium, a mixture of inorganic salts lacking a carbon source (Harris, 1989), to quantify the amount of variation among the isolates for growth characteristics. C. reinhardtii is normally grown photoautotrophically in Bold's medium, but it will also grow heterotrophically in the dark when the medium is supplemented with acetate as sole carbon and energy source. Its metabolism in the light is based on photosynthesis; in the dark it uses the glyoxalate shunt of the tricarboxylic acid cycle to provide both energy and carbon skeletons (Harris, 1989). These two environments therefore make very different metabolic demands on the organism and provide contrasting environments for selection, that we refer to as High and Dark. We also contrived one other treatment: Low, i.e. photoautotrophic growth in a 10\% Bold's solution, representing more nearly natural nutrient conditions.

Each of the 61 spores was inoculated from an agar slant into a culture tube containing Bold's medium and allowed to grow for seven days under continuous light. Subsequently, $25 \mu \mathrm{L}$ samples of these pre-inoculation cultures were used to inoculate $20 \mathrm{~mL}$ of growth medium in a culture tube of $1 \mathrm{~cm}$ internal diameter. Each spore was replicated twice. The inoculated culture tubes were placed in racks in a growth cabinet under constant illumination from six $100 \mathrm{~W}$ fluorescent lamps, situated $20 \mathrm{~cm}$ from the top of the tubes, in the case of the High and Low treatments. The Dark treatment was performed on adjacent covered shelves. The temperature was uncontrolled and varied between $25^{\circ} \mathrm{C}$ and $30^{\circ} \mathrm{C}$ during the experiments.

To analyse the variation among spores, growth was measured with a Bausch \& Lomb Spec-20D spectrophotometer at intervals of about two days, providing complete growth curves from which the logistic parameters $r$ and $K$, representing estimates of the growth capacity of spores, could be estimated (see Bell, 1991).

Data were analysed using the GLM procedure of SAS (1988). Normality and homogeneity of sampling variances were assessed. In the case of growth rate $(r)$ a logtransformation had to be used. The assays were laid out as complete unreplicated randomized blocks on each of two growth shelves per assay environment; the replication ('error') variance thus includes both within-shelf and among-shelf variance. Components of variance and covariance were estimated from the data, typically by equating observed and expected mean squares from analyses of variance (Falconer, 1982; Fry, 1992).

\section{Sorting}

We performed a competition-selection experiment to investigate the sorting of variation during the short-term of 50-70 generations. From each pre-inoculation culture, a $1-\mathrm{mL}$ sample was placed in a $250-\mathrm{mL}$ Erlenmeyer flask, to institute a more or less equal mixture of all 61 isolates, that we refer to as the founder assemblage or base population. We tested whether the mixture produced zygospores by growth on minimal medium (Harris, 1989), as an indication of sexual activity, but none were observed. Our experiment was therefore concerned with the sorting of variation among 61 vegetatively propagating strains. A $100-\mu \mathrm{L}$ sample of the vortexed founder assemblage was used to inoculate flasks representing different treatments. The High and Low lines were maintained in $300 \mathrm{~mL}$ of either High or Low environments in $500 \mathrm{~mL}$ Erlenmeyer flasks. The flasks were bubbled with filtered air under continuous fluorescent lighting. The Dark lines were maintained on the same growth shelves under identical conditions, except that the growth medium was supplemented with $1.2 \mathrm{~g} \mathrm{~L}^{-1}$ anhydrous sodium acetate and the flasks themselves were wrapped completely in aluminium foil to exclude light. The experiment was propagated by serial transfer of the asexual cultures for seven weeks. Each weekly transfer was initiated by inoculating $300 \mathrm{~mL}$ fresh medium with $100 \mu \mathrm{L}$ of old culture $\left(\approx 6 \times 10^{4}\right.$ cells), permitting $11-12$ doublings (to a final population size of roughly $2 \times 10^{8}$ cells) per cycle. In each environment, five replicate lines were established. The founder assemblage was stored on solid medium during the experiment by spreading $100 \mu \mathrm{L}$ of the original mixture on agar plates, also replicated five times, and stored in dim light to minimize growth. The essence of our experiment was the sorting of pre-existing variation in different environments by allowing competition among spores, while enforcing this process by weekly selection of the fastest growing spores through serial transfer to fresh media.

The High and Low lines went through seven growth cycles. The Dark lines went through four cycles. During the fifth growth cycle all cells died, apparently from starvation. Because the fourth-cycle flasks of the Dark selection lines had been stored, spores from these flasks were selected and stored for further usage. 
After finishing the experiment, four random spores were isolated from each selection line and from the stored lines of the founder assemblage. Each spore was assayed as described above. The selection and assay conditions were different (flasks and culture tubes), but growth in the two environments was positively correlated (Bell \& Reboud, 1997). The entire assay was blocked by replicating it in two growth cabinets. It thus comprised three selective environments (Dark, High and Low) $\times$ five lines $\times$ four spores per line $\times$ two replicates $\times$ three assay environments (Dark, High and Low $)=360$ cultures from the selection lines. The founder assemblage was treated in the same way, giving 120 cultures, representing the variation before selection.

To characterize changes in variation due to treatments, the data were analysed by factorial analysis of variance using the GLM procedure in SAS, to yield estimates of mean squares attributable to (assay) environment, lines, spores within lines, spore-by-environment interaction and replication (error).

\section{Results}

\section{Initial diversity}

Repeatabilities in single environments ranged from 0.32 to 0.68 (Table 1), indicating substantial variation for growth characteristics when the spores were grown as pure cultures in an uniform environment. Thirteen of the 61 tested spores did not display heterotrophic growth, at least not with acetate as a carbon source. The most conspicuous feature from the combined analysis is the large contribution made by the sporeby-environment interaction (Table 1). This is most simply expressed by the intraclass correlation coefficient

$t_{\mathrm{S}}=\sigma_{\mathrm{S}}^{2} /\left(\sigma_{\mathrm{S}}^{2}+\sigma_{\mathrm{SE}}^{2}\right)$

where $\sigma_{\mathrm{S}}^{2}$ is the among-spore variance and $\sigma_{\mathrm{SE}}^{2}$ the spore-by-environment interaction. This will be close to unity if the performance of spores is highly consistent across environments, but close to zero if relative performance is nearly independent in different environments. The values of $t_{\mathrm{S}}$ for growth rate $(0.34)$ and carrying capacity $(0.23)$ are comparable with previous experiments (Bell, 1990, 1991) and confirm that sporeby-environment interaction makes the predominant contribution to the overall spore variance of growth characteristics.

The correlations between growth in the dark and growth in any light environment were very low (range: -0.19 to $0.26, n=48$ or 61 ). This is consistent with the extensive spore-by-environment interaction observed. Growth in High and Low environments was positively correlated for growth rate $(r=0.35, P<0.05)$. The correlation between the two growth measures $r$ and $K$ was positive in the Dark environment $(r=0.26, \mathrm{NS})$ and negatively correlated in both High $(r=-0.40, P<0.05)$ and Low $(r=-0.49, P<0.01)$ environments, indicating that the relationship between growth measures varies among environments.

Table 1 Variance components $\uparrow$ (based on a one-way ANOva within each environment) and repeatabilities of growth parameters $r\left(\right.$ day $\left.^{-1}\right) ; K$ (maximum density) in populations of unicellular green soil algae

\begin{tabular}{|c|c|c|c|c|c|c|}
\hline \multirow[b]{2}{*}{ Assay environment } & \multicolumn{3}{|c|}{ Growth rate $(r)$} & \multicolumn{3}{|c|}{ Carrying capacity $(K)$} \\
\hline & Dark & High & Low & Dark & High & Low \\
\hline (Among) Spores & 0.0015 & 0.0062 & 0.0172 & 4081 & 3145 & 456 \\
\hline (Among) Replicates & 0.0032 & 0.0038 & 0.0334 & 2934 & 1917 & 900 \\
\hline Repeatability & 0.32 & 0.62 & 0.34 & 0.58 & 0.62 & 0.34 \\
\hline $\begin{array}{l}\text { Combined ANOVA: } \\
\text { Source }\end{array}$ & d.f. & MS & $\mathrm{VC}$ & & MS & $\mathrm{VC}$ \\
\hline Assay environment & 2 & 39.079 & - & & 2821436 & - \\
\hline Spores & 47 & 0.104 & 0.012 & & 5650 & 685 \\
\hline Assay $\times$ spores & 94 & 0.085 & 0.023 & & 7526 & 2282 \\
\hline Replicates & 140 & 0.037 & 0.037 & & 1720 & 1721 \\
\hline
\end{tabular}

$\dagger$ Variance components in the single environments are: among-spores (d.f. $=48$ or 61$)$ and among-replicate cultures of the same spore (d.f. $=48$ or 61). All among-spores components were significant at $P<0.05$ (most at $P<0.01$ ). Different degrees of freedom are caused by failure of spores to grow in the dark. For the combined analysis the mean squares (MS) and variance components (VC) of the random factors are given. Effects tested are: among assay environment, among-spore, and assay-by-spore interaction. All effects were significant at $P<0.001$.

(C) The Genetics Society of Great Britain, Heredity, 87, 162-171. 


\section{Sorting}

The mean and variance of growth parameters before and after applying the treatments are given in Table 2. There was substantial variation among spores in the founding assemblage. This was greater when assayed in the dark then when assayed in the light: all lines grew well in the light, but some lines were scarcely able to grow in the dark, whereas others grew almost as well in the dark as in the light.

Figure 1 shows the response to different treatments with respect to $r$ and $K$. Differences between founder and selected populations were in general more pronounced for $K$ than for $r$, especially in the light treatments. Differences between founder and selected population were tested by a nested ANOvA with the among-lines mean square as the error term. Consequently, only few degrees of freedom are available per test (d.f. $=1,8$ for $F$-tests), and the results therefore indicate large shifts in environment-specific growth (Table 2). The Dark and High treatments greatly reduced the among-spore variation initially present in the founder assemblage, and most of the spores extracted from the cultures at the end of the experiment were equivalent to the fastest growing isolates of the initial mixture (Fig. 1). This was less obvious in the Low treatment, where the among-spore variance was still substantial, and the population means had hardly changed. In the Dark treatment no amongspore variation was left for either $r$ or $K$ (Table 3 ). In the High treatment there was still some variation remaining, attributable largely to a significant amonglines and assay-environment-by-line interaction variance. In the Low treatment, variance after selection was somewhat reduced but still substantial (Fig. 2; Table 3). In the Dark treatment there was also a large reduction of the assay-environment-by-spore interaction variance and an increase in the environmental variance (Fig. 2). Fifty generations of sorting had apparently reduced the diverse original assemblage, comprising more than 60 different spores, to a single specialized spore, at least based on the phenotypic characteristics.

\section{Relationship between advance and regress}

About 50-70 generations of selection were sufficient to produce marked divergence among the lines for both growth parameters (Fig. 3). The Dark and High treatments are negatively correlated, i.e. spores that grew well in the dark grew less well in the light, and vice versa. Advance or regress in the dark was much more pronounced than in the light. The two light treatments, High and Low, were positively correlated. Thus, when using contrasting environments, advance in the selection environment was associated with regress in other environments.

\section{Discussion}

There were two features of these results which seem likely to be of general interest: the high diversity with respect to components of growth in communities of soil algae; and the rapid sorting of the algal community during short-term selection in different laboratory environments.

\section{Diversity}

We found a great deal of variation in growth rate among spores collected a few metres apart. The magnitude of the spore-by-environment interaction variance is especially striking, indicating that growth rate changes over environments and is highly sensitive to particular combinations of environmental factors. This might be taken to imply that the wheat field, although regularly ploughed, harrowed and fertilized, and homogeneous to the eye, is in fact heterogeneous with respect to conditions of growth. This was also reflected in the spatial organization of the community, because variance of growth measures increased only slowly with distance (results not shown) indicating that nearby sites are poorly correlated and that the spores are likely to encounter a range of conditions (see Bell et al., 1993).

Although our scale of sampling was one metre, we consider it likely that environmental and among-spore variance exists at scales of millimetres or centimetres. A sampling interval of one metre might be too large with respect to the dispersal capacities of soil algae. Most algae stick to the sand and clay grains and do not seem to disperse actively, thereby exploiting very small-scale spatial variation. We intend to investigate this possibility in the future.

One might argue that these organisms do not usually grow heterotrophically in the dark and that the sporeby-environment interaction measured in the laboratory overestimates that expressed in the field. Omitting this treatment, however, and using only the High and Low treatment does not change the conclusion, although the effects are less pronounced. Thus, quantitative differences in nutrient availability might alone support specialization. One way of investigating this issue would be to culture isolates in aqueous extracts of soil samples from which they were collected, in order to measure the extent of local adaptation at a given spatial scale. 
Table 2 Mean and variance $(n=40)$ of growth parameters of founder and selected populations of unicellular green soil algae when grown in three distinctive environments (Dark, High Bold's and Low Bold's); (a) growth rate (r); (b) carrying capacity $(K)$

(a)

\begin{tabular}{|c|c|c|c|c|c|c|}
\hline \multirow[b]{3}{*}{ Selective environment } & \multicolumn{6}{|c|}{ Growth rate $(r)$} \\
\hline & \multirow[b]{2}{*}{ Mean } & \multirow[b]{2}{*}{ Variance } & \multirow[b]{2}{*}{ Ratio $\dagger$} & \multicolumn{3}{|c|}{ Variance components } \\
\hline & & & & Lines & Spores & Error \\
\hline \multicolumn{7}{|l|}{ Dark assay } \\
\hline Founder & 0.848 & 0.127 & & 0 & $0.223 * * *$ & 0.007 \\
\hline Dark & 1.277 & 0.013 & $1.51 * * *$ & 0.001 & 0 & 0.008 \\
\hline High & 0.705 & 0.016 & 0.830 .08 & 0.009 & $0.016^{* *}$ & 0.012 \\
\hline Low & 0.915 & 0.126 & $1.08 \mathrm{NS}$ & 0.021 & $0.129 * * *$ & 0.004 \\
\hline \multicolumn{7}{|l|}{ High Bold's assay } \\
\hline Founder & 0.604 & 0.038 & & 0.012 & $0.109 *$ & 0.094 \\
\hline Dark & 0.507 & 0.049 & 0.840 .07 & 0.013 & 0.008 & 0.127 \\
\hline High & 0.732 & 0.061 & $1.21 \mathrm{NS}$ & $0.054 * *$ & 0.021 & 0.041 \\
\hline Low & 0.635 & 0.032 & $1.05 \mathrm{NS}$ & $0.027^{*}$ & 0.002 & 0.055 \\
\hline \multicolumn{7}{|l|}{ Low Bold's assay } \\
\hline Founder & 0.846 & 0.058 & & 0 & $0.043^{*}$ & 0.048 \\
\hline Dark & 0.719 & 0.057 & $0.85^{*}$ & 0 & 0.014 & 0.077 \\
\hline High & 1.005 & 0.049 & $1.19^{*}$ & 0.005 & 0.001 & 0.043 \\
\hline Low & 0.900 & 0.045 & $1.06 \mathrm{NS}$ & $0.021 *$ & 0.011 & 0.022 \\
\hline
\end{tabular}

(b)

\begin{tabular}{|c|c|c|c|c|c|c|}
\hline \multirow[b]{3}{*}{ Selective environment } & \multicolumn{6}{|c|}{ Carrying capacity $(K)$} \\
\hline & \multirow[b]{2}{*}{ Mean } & \multirow[b]{2}{*}{ Variance } & \multirow[b]{2}{*}{ Ratio } & \multicolumn{3}{|c|}{ Variance components } \\
\hline & & & & Lines & Spores & Error \\
\hline \multicolumn{7}{|l|}{ Dark assay } \\
\hline Founder & 680 & 10130 & & 0 & $11298 * * *$ & 681 \\
\hline Dark & 772 & 807 & $1.14 * *$ & 125 & 253 & 272 \\
\hline High & 599 & 2440 & $0.88 * *$ & 0 & $1671^{* *}$ & 912 \\
\hline Low & 693 & 5349 & $1.01 \mathrm{NS}$ & 0 & $6487 * * *$ & 435 \\
\hline \multicolumn{7}{|l|}{ High Bold's assay } \\
\hline Founder & 570 & 29750 & & 0 & $31149 * * *$ & 4070 \\
\hline Dark & 338 & 4508 & $0.59 * * *$ & 0 & 2185 & 2929 \\
\hline High & 729 & 3329 & $1.28 * * *$ & 0 & $2192 * * *$ & 1132 \\
\hline Low & 708 & 10842 & $1.24 *$ & 0 & $7011 * * *$ & 2480 \\
\hline \multicolumn{7}{|l|}{ Low Bold's assay } \\
\hline Founder & 406 & 7333 & & 0 & $7659 * * *$ & 1417 \\
\hline Dark & 265 & 5416 & $0.65^{* * *}$ & 0 & 490 & 5215 \\
\hline High & 476 & 1298 & $1.17 * * *$ & 92 & 399 & 773 \\
\hline Low & 452 & 1883 & $1.11 * *$ & 114 & $968^{* *}$ & 766 \\
\hline
\end{tabular}

$\dagger$ 'Founder' is stored material of spores used to found each selection line. Variance components are based on a nested ANOVA: among lines $($ d.f. $=4)$, among spores within lines $($ d.f. $=15)$ and among replicate cultures of the same spore $($ d.f. $=20)$. Estimates of variance components were obtained by equating observed with expected mean squares. Negative estimates have been set to zero. 'Ratio' represents the relative fitness, i.e. selected/founder population and the significance level of a test for the difference between founder and selected populations based on a nested ANOvA with the among-lines mean square as the error term.

${ }^{*} P<0.05 ; * * P<0.01 ; * * * P<0.001$.

(C) The Genetics Society of Great Britain, Heredity, 87, 162-171. 


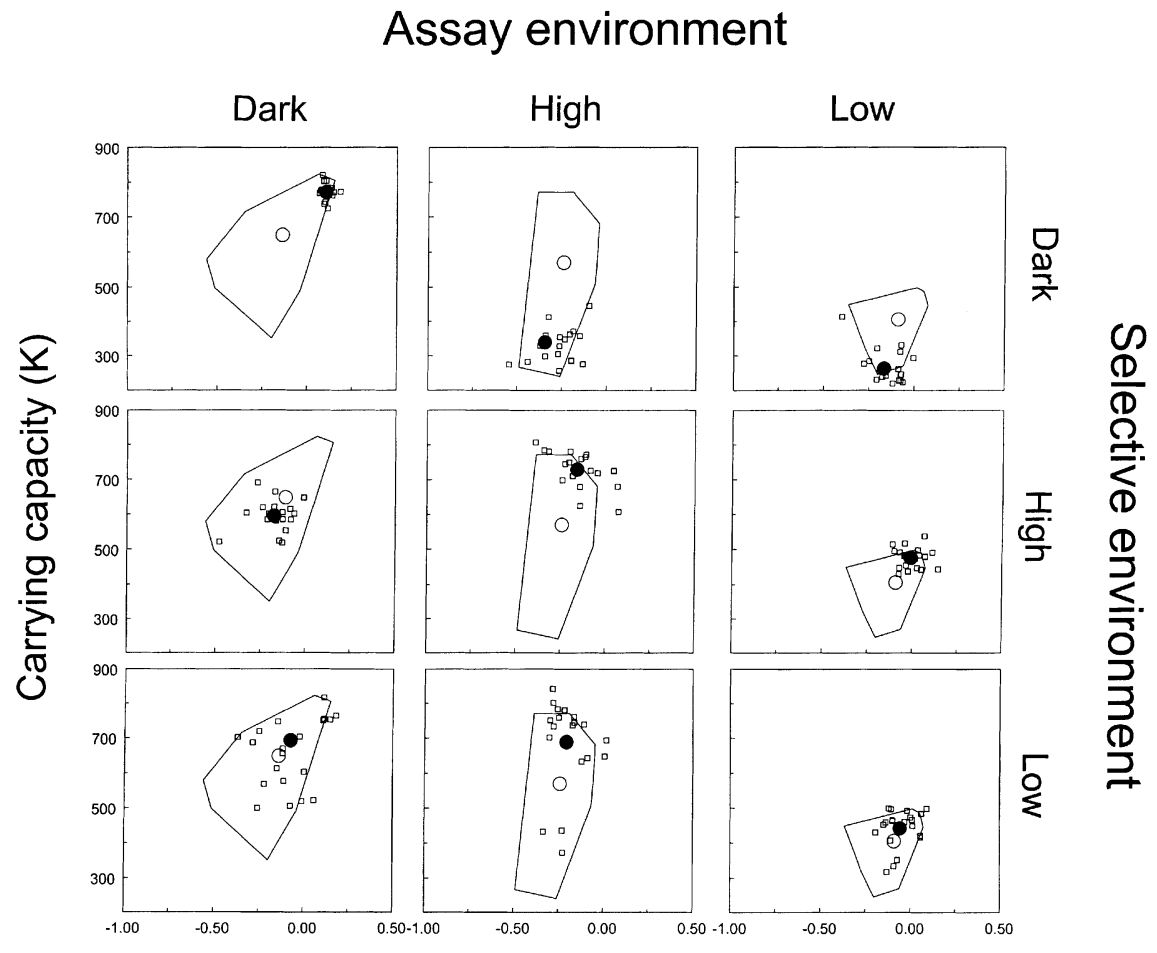

(log) Growth rate (r)
Fig. 1 Performance of spores from lines selected in different environments (Dark, High Bold's, and Low Bold's) in three assay environments (Dark, High Bold's, and Low Bold's): phenotypes after selection. Data are shown as a plot of the $(\log )$ growth rate $r$ vs. the carrying capacity $K$. The envelope represents the variation in the founder or base population from which the selection experiment started. Each square $(\square)$ is the mean of two replicates of each spore. The average of all spores before and after selection is indicated by a hollow $(\bigcirc)$ or solid circle $(\mathbf{O})$, respectively.

Table 3 Factorial analysis of variance for the logistic growth parameters in the sorting experiment in populations of unicellular green soil algae

\begin{tabular}{|c|c|c|c|c|c|c|}
\hline Selective environment & Model & d.f. & Founder $\mathrm{MS}^{\dagger}$ & Dark MS & High MS & Low MS \\
\hline \multicolumn{7}{|l|}{ Carrying capacity $(K)$} \\
\hline 1 Assay environment & $1 / 4$ & 2 & $69.82 *$ & $296.17 * * *$ & $63.78 *$ & $69.94 * * *$ \\
\hline 3 Spores within lines & $3 / 6$ & 15 & $2.65 * * *$ & 0.63 & $0.28 * *$ & $0.44 * * *$ \\
\hline 4 Assay $\times$ line & $4 / 5$ & 8 & 1.22 & 0.28 & 0.39 & 1.38 \\
\hline 5 Assay $\times$ spore & $5 / 6$ & 30 & $4.20 * * *$ & 0.36 & $0.42 * * *$ & $1.51 * * *$ \\
\hline \multicolumn{7}{|l|}{ Growth rate $(r)$} \\
\hline 1 Assay environment & $1 / 4$ & 2 & $1.245^{*}$ & $10.037 * * *$ & $1.592 *$ & 1.318 \\
\hline 2 Lines & $2 / 3$ & 4 & 0.223 & 0.221 & $0.219 *$ & 0.130 \\
\hline 3 Spores within lines & $3 / 6$ & 15 & $0.179 * *$ & 0.098 & 0.052 & $0.132 * *$ \\
\hline 4 Assay $\times$ line & $4 / 5$ & 8 & 0.195 & 0.080 & $0.278 * *$ & $0.403 * *$ \\
\hline
\end{tabular}

$\dagger$ Expected mean squares (MS) are based on a mixed model with assay environment (Dark, High Bold's and Low Bold's) as fixed and lines and spores within lines as random effects. Model indicates the error term over which an effect is tested. Significance levels as in Table 2.

\section{Sorting}

Our initial assemblage of freshly extracted Chlamydomonas-like soil algae displayed both a significant among-spore variance as well as a substantial amount of spore-by-environment interaction. In the two most contrasting environments, Dark and High, there was a clear treatment response, that took the selected population to the limits of the phenotypic variation present in the founder assemblage (Fig. 1). The time scale of the experiment (50-70 generations) is therefore appropriate for investigating the short-term effect of selection in 
Fig. 2 Response of among-spore, environmental and spore-by-environment interaction variances to selection for the logistic growth parameters (log) growth rate $(r)$ and carrying capacity $(K)$.

Variance components were derived from the analyses of variance in Table 3

(B, Founder population; D, selection in the dark; H, selection in high Bold's; L, selection in low Bold's).
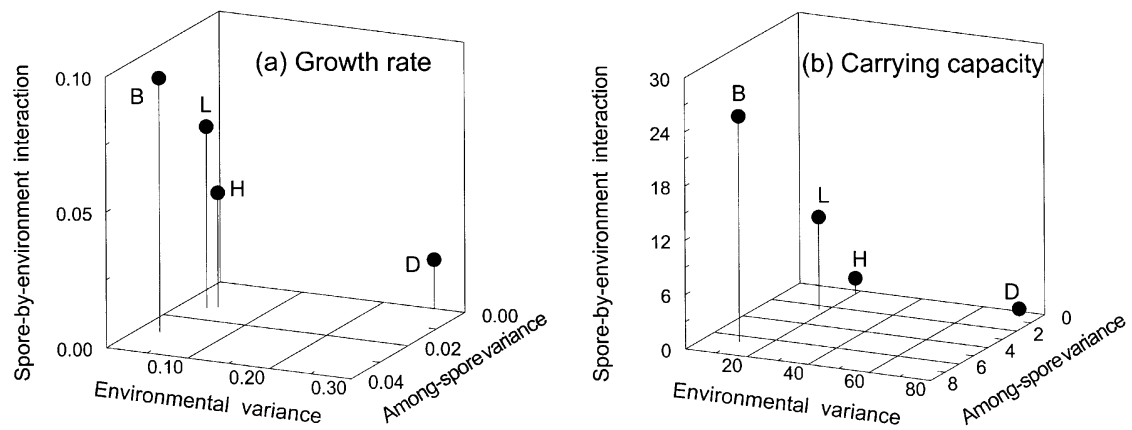

Growth rate

Carrying capacity

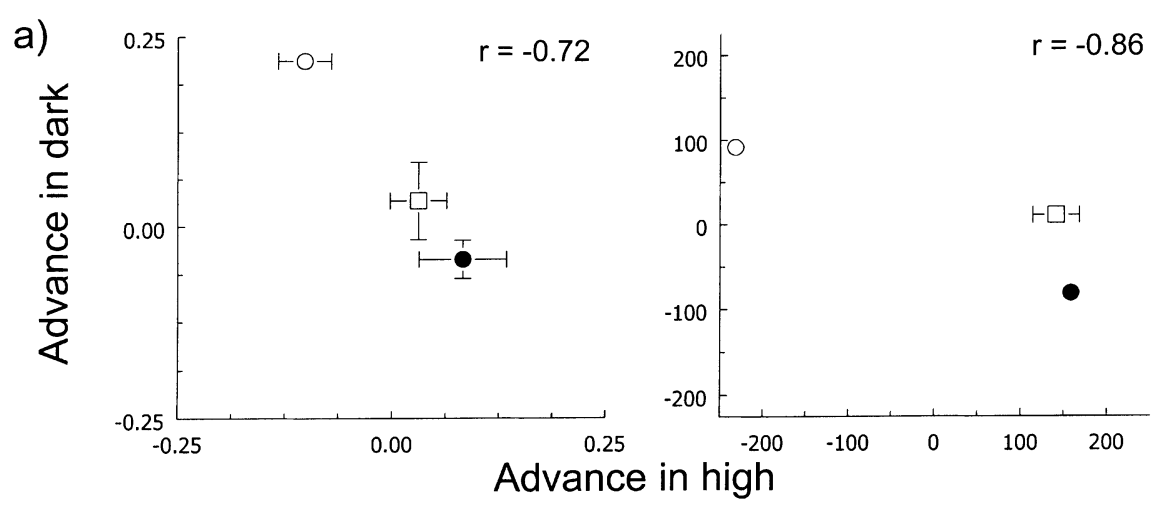

b)

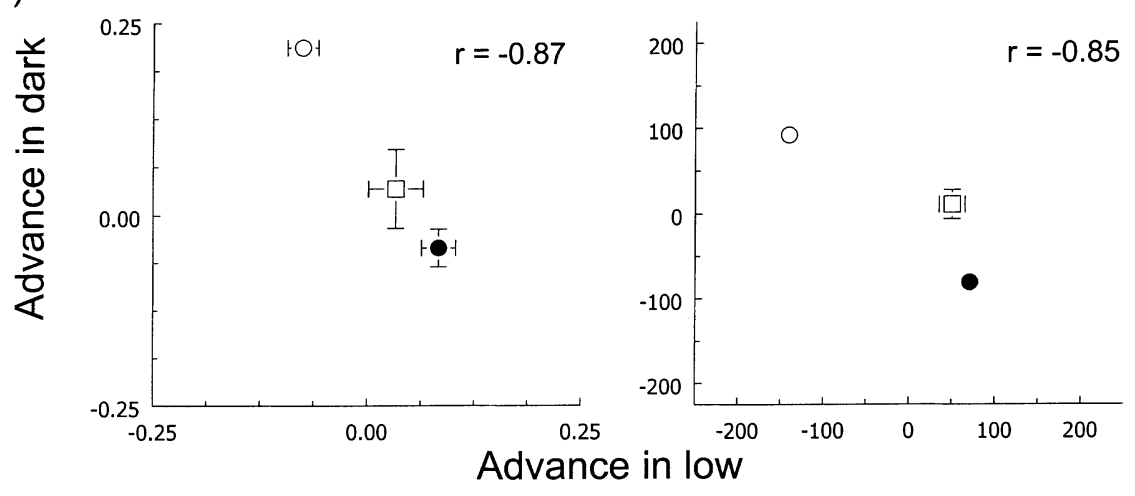

c)

Fig. 3 Relationship between advance logistic growth parameters. Mean values ( $\pm \mathrm{SE}, n=5$ lines) of the selective environments relative to the founder population: (a) advance in high Bold's vs. advance in the dark; (b) advance in low Bold's vs. advance in the dark; (c) advance in high Bold's vs. advance in low Bold's ( $\bigcirc$ selection in the dark, selection in high Bold's, $\square$ selection in low Bold's). Also shown are the correlations between advance and regress ( $n=15$ line means, all correlations significant at at least the $P<0.01$ level).
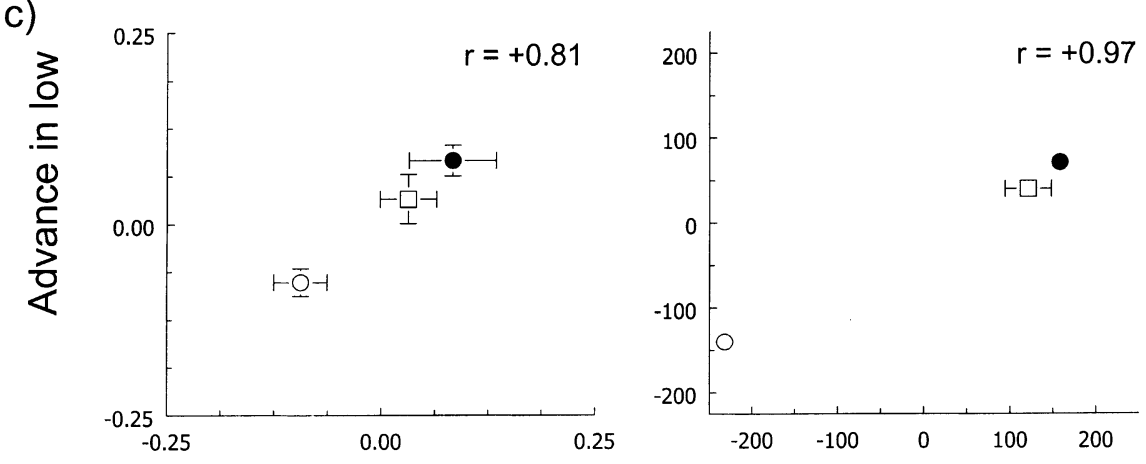

Advance in high

(C) The Genetics Society of Great Britain, Heredity, 87, 162-171. 
sorting the variation initially present in a diverse population. The reduction or elimination of amongspore variation in growth characteristics under selection in these uniform environments is expected from first principles. Nevertheless, it does not necessarily imply the loss of all diversity: several or many types might be preserved, provided that they express similar phenotypes in the selection environment, even though they might express different phenotypes in other environments. In this experiment, no among-spore variance appeared in the Dark selected spores for either $r$ or $K$ when they were tested in either High or Low environments (Table 2) and no spore-by-environment interaction was detected (Fig. 2; Table 3), suggesting the existence of a single type. In contrast, the High selected spores still displayed among-spore variance for $K$ when tested in either the High, Low or Dark environment (Table 2). No amongspore variance was left for $r$ when tested in the High environment, but significant among-spore variance was detected for these spores in the Dark environment (Table 2). Thus, the phenotypically uniform population after selection in its own environment still displayed variation in other environments. It is quite possible, of course, that these spores did vary somewhat in growth rate, so that continued selection would eventually produce true uniformity.

The continuous sorting of variation in strongly contrasting environments created a negative correlation for both maximal growth rate $(r)$ and carrying capacity $(K)$. This will be due to the selection for types specifically adapted to the particular circumstances. The correlated response was either antagonistic or absent. When the difference between the environments was less pronounced, positive correlated responses were also observed. Thus the antagonism between the performance in different environments was a function of the difference between these environments.

The behaviour of the replicate lines varied among treatments. In the Dark environment, all lines responded in the same way and there was little variation between them. In the High and Low treatments, however, replicate lines differed substantially after finishing the experiment. They sometimes responded in opposite ways, and in one case the response of one of the Low lines even exceeded that of the extreme environments, giving rise to a highly adapted line. Even when all lines are inoculated from the same mixture, random processes can cause divergence among replicates when selection is relatively weak.

\section{Diversity of soil microbes}

The biodiversity of the microbes and microfauna of the soil is very difficult to study by conventional techniques. The identification of formal taxa is often difficult, and requires expertise that is often in very short supply. Molecular approaches are being developed, but they are still laborious and expensive, and require arbitrary judgement of the significance of sequence variation (Hill et al., 1995; Von Wintzingerode et al., 1997). The phenotypic approach that we have chosen does not provide information about the number of discrete types in a volume of soil, but instead demonstrates the presence and magnitude of genetic variance for growth in different conditions through a selection experiment. The rapid response of the community shows that it did indeed contain a range of different kinds adapted to different conditions of growth. This is a technique that can readily be applied to large numbers of isolates from any kind of habitat, and might provide a useful adjunct to conventional or molecular taxonomy in evaluating levels of microbial diversity.

\section{Acknowledgements}

We thank C. Potvin and family for permitting us to sample their fields. Research supported by NSERC (Canada) operating grant to G. Bell and NWO (Netherlands Organization for Scientific Research) postgraduate scholarship to H. P. Koelewijn.

\section{References}

ANTONOVICS, J. AND LEVIN, D. A. 1980. The ecological and genetic consequences of density-dependent regulation in plants. Ann. Rev. Ecol. Syst., 11, 411-452.

BALL, D. F. AND WILliams, w. M. 1968. Variability of soil properties in two uncultivated brown earths. J. Soil Sci., 19, 379-391.

BECKETT, P. H. T. AND WEBSTER, R. 1971. Soil variability: a review. Soils Fertilizers, 34, 1-15.

BeLL, G. 1990. The ecology and genetics of fitness in Chlamydomonas. I. Genotype-by-environment interactions among pure strains. Proc. Royal Soc. B, 240, 295-321.

BELL, G. 1991. The ecology and genetics of fitness in Chlamydomonas. III. Genotype-by-environment interactions within strains. Evolution, 45, 668-679.

BELL, G. 1997. Experimental evolution in Chlamydomonas. I. Short-term selection in uniform and diverse environments. Heredity, 78, 490-497.

BELL, G. AND REBOUD, X. 1997. Experimental evolution in Chlamydomonas II. Genetic variation in strongly contrasted environments. Heredity, 78, 498-506.

BELL, G., LECHOWICZ, M. J., APPENZELLER, A., CHANDLER, M. ET AL. 1993. The spatial structure of the physical environment. Oecologia, 96, 114-121.

BENNETT, A. F. AND LENSKI, R. E. 1993. Evolutionary adaptation to temperature. II. Thermal niches of experimental lines of Escherichia coli. Evolution, 47, 1-12.

BENNETT, A. F., LENSKI, R. F. AND MITTLER, J. E. 1992. Evolutionary adaptation to temperature. I. Fitness responses 
of Escherichia coli to changes in its thermal environment. Evolution, 46, 16-30.

FALCONER, D. S. 1982. Introduction to Quantitative Genetics, 2nd edn. Longman, Harlow, England.

FRY, J. D. 1992. The mixed-model analysis of variance applied to quantitative genetics: biological meaning of the parameters. Evolution, 46, 540-550.

GILlESPIE, J. H. AND TURELLI, M. 1989. Genotype-by-environment interactions and the maintenance of polygenic variation. Genetics, 121, 129-138.

HARRIS, E. 1989. The Chlamydomonas Source Book. Academic Press, New York.

HEDRICK, P. W. 1986. Genetic polymorphism in heterogeneous environments: a decade later. Ann. Rev. Ecol. Syst., 17, 535-566.

HEYwood, J. S. 1991. Spatial genetic analysis of genetic variation in plant populations. Ann. Rev. Ecol. Syst., 22, 335-355.

HILL, G. T., MITKOWSKI, N. A., ALDRICH-WOLFE, L., EMELE, L. R. ET AL. 1995. Methods for assessing the composition of soil microbial communities. Appl. Soil Ecol., 15, 25-36.

KIBOTA, T. T. AND LYNCH, M. 1996. Estimation of the genomic mutation rate deleterious to overall fitness in E. coli. Nature, 381, 694-696.

LECHOWICZ, M. L. AND BELL, G. 1991. The ecology and genetics of fitness in forest plants. II. Microscale heterogeneity of the edaphic environment. J. Ecol., 79, 686-696.

LENSKI, R. E. AND TRAVISANO, M. 1994. Dynamics of adaptation and diversification: a 10,000-generation experiment with bacterial populations. Proc. Nat. Acad. Sci. U.S.A., 91, 6808-6814.
LEVENE, H. 1953. Genetic equilibrium when more than one ecological niche is available. Am. Nat., 87, 131-133.

LEVINS, R. 1968. Evolution in Changing Environments. Princeton University Press, Princeton, NJ.

REBOUD, X. AND BELL, G. 1997. Experimental evolution in Chlamydomonas. III. Evolution of specialist and generalist types in environments that vary in space and time. Heredity, 78, 507-514.

rosenzweig, M. 1995. Species Diversity in Space and Time. Cambridge University Press, Cambridge.

SACK, L., ZEYL, C., BELl, G., ShARBel, T. ET AL. 1994. Four new strains of Chlamydomonas reinhardtii. J. Phycol., 30, 770-773.

SAS InSTITUTE. 1988. SAS Statistics Handbook. Cary, NC.

TAYLOR, A. A., DE-FELICE, J. J. AND HAVILL, C. D. 1982. Seasonal variation in nitrogen availability and utilization in an acidic and calcareous soil. New Phytol., 92, 141-152.

TRAVISANO, M., MONGOLD, J. A., BENNETT, A. F. AND LENSKI, R. F. 1995. Experimental tests of the role of adaptation, chance and history in evolution. Science, 267, 87-90.

TROELSTRA, S. R., LOTZ, L. A. P., WAGENAAR, R. AND SLUiMER, L. 1990. Temporal and spatial variability in soil nutrient status of a former beach plain. Plant and Soil, 127, 1-12.

VON WINTZINGERODE, F., GOEBEL, E. B. AND STACKEBRANDT, E. 1997. Determination of microbial diversity in environmental samples: pitfalls of PCR-based rRNA analysis. FEMS Microbiol. Rev., 21, 213-229.

westerman, R. L. 1990. Soil Testing and Plant Analysis. Soil Science Society of America, Madison, WI. 